\title{
Pre-exposure to context affects learning strategy selection in mice
}

\author{
Tumay Tunur, ${ }^{1}$ Gary P. Dohanich, ${ }^{2,3}$ and Laura A. Schrader ${ }^{1,3,4}$ \\ ${ }^{1}$ Department of Cell and Molecular Biology, Tulane University, New Orleans, Louisiana 70118, USA; ${ }^{2}$ Department of Psychology, \\ Tulane University, New Orleans, Louisiana 70118, USA; ${ }^{3}$ Program in Neuroscience, Tulane University, New Orleans, \\ Louisiana 70118, USA
}

\begin{abstract}
The multiple memory systems hypothesis proposes that different types of learning strategies are mediated by distinct neural systems in the brain. Male and female mice were tested on a water plus-maze task that could be solved by either a place or response strategy. One group of mice was pre-exposed to the same context as training and testing (PTC) and the other group was pre-exposed to a different context (PDC). Our results show that the PTC condition biased mice to place strategy use in males, but this bias was dependent on the presence of ovarian hormones in females.
\end{abstract}

The participation of different brain areas in place and response learning strategies has been studied extensively (White and McDonald 2002; Gold 2004; Mizumori et al. 2004). Place strategy is an allocentric navigation strategy that depends on extramaze cues. Response strategy is an egocentric navigation strategy based on proprioceptive cues. Inactivation of the hippocampus biased animals to response strategy use, and inactivation of the striatum biased animals to place strategy use (Packard and McGaugh 1996; Lee et al. 2008). Furthermore, glutamate infusion into the hippocampus strengthened place strategy use and, conversely, glutamate infusion into the striatum enhanced response strategy use (Packard 1999). These studies suggest that the hippocampus system mediates place strategy, while the striatum system mediates response strategy.

Various factors can modulate learning strategy use, including training intensity (Packard and McGaugh 1996; Martel et al. 2007). A recent study investigated the influence of training on strategy use on a probe trial conducted $1 \mathrm{~h}$ after training (Martel et al. 2007). Male mice displayed enhanced place strategy use when trained on 12 or 22 trials compared with four trials, suggesting an effect of training intensity on strategy choice (Martel et al. 2007). This study further investigated the effect of pre-exposure to the training and testing context (PTC). Pre-exposure enhanced place strategy use in male mice after only four trials relative to animals pre-exposed to a different context (PDC). These results suggest that a sufficient exposure to the training and testing context promotes place strategy use in mice.

The type of strategy used by rats is affected by both biological sex and gonadal steroids. Male rats typically employ a place strategy, especially during the early phase of training, on both land and water T-mazes (Packard and McGaugh 1992, 1996; Packard and Teather 1997). However, strategy use by female rats depends on hormonal conditions (Dohanich 2002; Dohanich et al. 2009). Place strategy is preferred by intact female rats on the day of proestrus when estradiol levels are elevated, and by ovariectomized rats treated with estradiol (Korol and Kolo 2002; Korol 2004; Korol et al. 2004). In contrast, response strategy is more often displayed by intact females on diestrus, and by ovariectomized females that did not receive estradiol replacement (Korol and Kolo 2002; Korol et al. 2004). To date, the effects of biological

\footnotetext{
${ }^{4}$ Corresponding author.
}

E-mail schrader@tulane.edu; fax (504) 865-6785.

Article is online at http://www.learnmem.org/cgi/doi/10.1101//m.1818410. sex and gonadal steroids on learning strategy have not been studied in mice.

In this study, we developed a modified version of the dualsolution water plus-maze task to further investigate the role of PTC compared with PDC in male and female mice. We hypothesized that strategy choice in both sexes would be dependent on context pre-exposure, and ovarian hormones would influence strategy choice in females. Our results show that PTC significantly enhanced place strategy use in male mice. Although there was no significant difference between PTC and PDC female mice, ovariectomy significantly reduced place strategy use in the PTC females, suggesting that ovarian hormones play a significant role in strategy use in female mice.

Sixteen male and 39 female 129/Sve strain mice were obtained at 2-3 mo of age from Charles River Laboratories (Boston, MA). Mice were housed in groups of four on a 12/12 light/dark cycle (lights on at 07:00 h) with free access to food and water. All protocols followed the guidelines from a protocol approved by the Animal Care and Use Committee of Tulane University in accordance with National Institutes of Health Guide for the Care and Use of Laboratory Animals.

Mice were pre-exposed for $5 \mathrm{~min}$ to the dry plus-maze either in the context of the subsequent training and testing (PTC), or in a different context in a different room (PDC), $30 \mathrm{~min}$ prior to the first training trial. The maze consisted of four clear Plexiglas arms ( $40 \mathrm{~cm}$ in length, $10 \mathrm{~cm}$ in width, and $40 \mathrm{~cm}$ in height). During the pre-exposure, mice were able to visit three arms of the maze. The rooms had different visual cues surrounding the maze. No extramaze cues were placed directly at the end of any arm. After the pre-exposure, the animal was placed in its home cage. The maze was wiped clean with $70 \%$ ethanol between trials.

After pre-exposures, the maze was filled to $1.5 \mathrm{~cm}$ above the Plexiglas escape platform $(15 \mathrm{~cm}$ in height $)$ with roomtemperature water colored opaque with white nontoxic tempera paint. Mice were trained in the water plus-maze task (Fig. 1A). The training was ended when the animals made six correct choices or reached nine trials. The animals that made fewer than four correct choices during training were not included in the study. Trials were continued until the mouse reached the platform or a maximum of $1 \mathrm{~min}$. Each trial was separated by an intertrial interval of $4 \mathrm{~min}$. Throughout the training trials, one arm (north) was blocked off by a white Plexiglas shield, creating a T-shaped maze. Mice were placed in the start arm of the maze (south) and were allowed to swim to the escape platform, which 

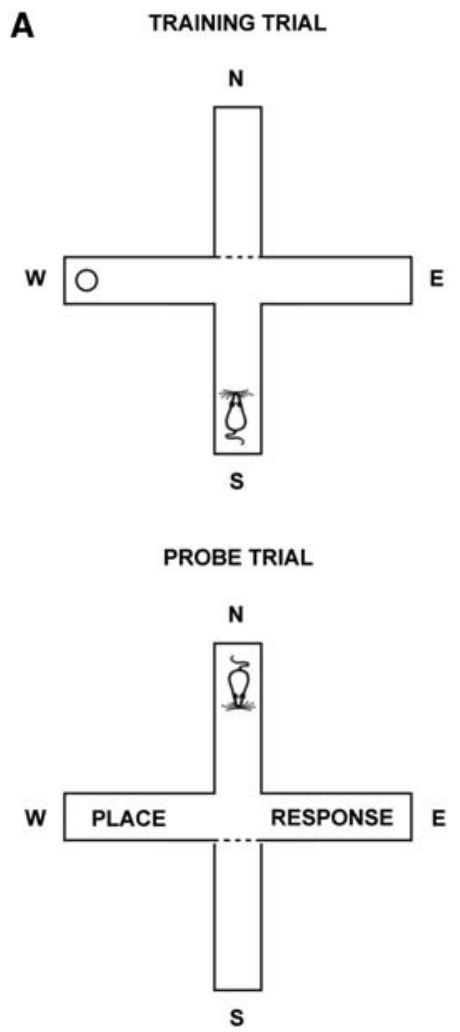

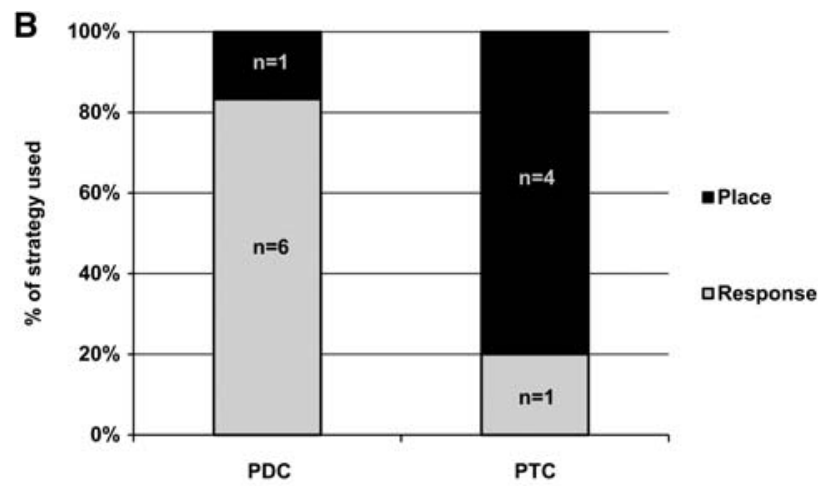

C Latency Curves (Males)

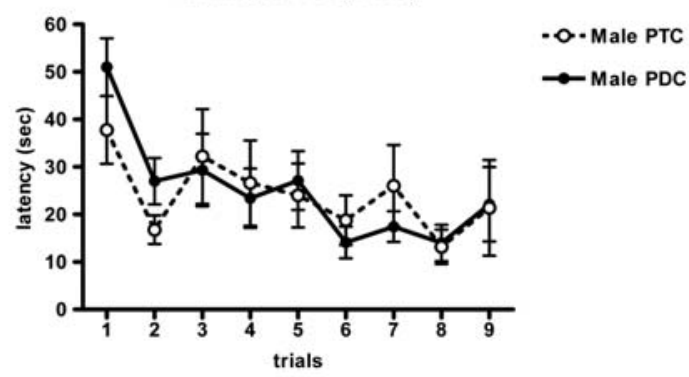

Figure 1. The effects of pre-exposure to the training and testing context (PTC) or to a different context (PDC) on strategy selection of male mice. (A) Schematic diagram of the water plus-maze. Mice were released from the south arm during training trials and from the north arm during the probe test. (B) More male mice used place strategy than response strategy when pre-exposed to the same context prior to training and testing (PTC, $n=5$ ) compared with male mice pre-exposed to a different context (PDC, $n=7, P<0.05$ ). (C) Latency curves show the actual latency to escape to the platform. Two-way ANOVA (non-repeated measures) revealed no significant difference across training trials in escape latencies between PDC and PTC mice $(P>0.5)$, although a significant effect of trial indicated that mice reduced their escape latencies across trials $(P<0.001)$. Values represent mean \pm S.E.M.

was consistently located in one arm of the maze for each animal and alternated between animals (east or west). Entry of the entire animal into the maze arm that contained the escape platform was scored as a correct response during the training trials, and entry of the entire animal into the maze arm that did not contain the escape platform was scored as an incorrect response. Mice were allowed to remain on the escape platform for $15 \mathrm{sec}$ before being returned to their cages. Mice that failed to find the escape platform within $60 \mathrm{sec}$ were manually guided to the platform. The water was distributed across all arms of the maze and the maze walls were wiped down to reduce intramaze cues between training and probe trials. One hour after training, mice were tested on a probe trial (Fig. 1B) in order to determine their relative use of "place" and "response" strategy. On the probe trial, mice were placed into the start arm $180^{\circ}$ opposite the start arm used during training (i.e., end of the north arm) and were allowed to make an entry into either the east or west maze arm. The white Plexiglas shield blocked the south arm during the probe trial. Mice were designated as using place or response strategy based on the probe trial. Place strategy was designated as entry of the entire animal into the arm with the platform, and response strategy was designated as entry of the entire animal into the opposite arm.

Sixteen male mice were randomly divided into two groups based on pre-exposure context, PTC or PDC. Four of the 16 males were not included in the study for failure to reach criterion (four correct out of nine trials) or failure to escape to the platform due to floating, which is a behavior commonly seen in this strain
(Wolfer et al. 1997). On the probe trial PTC males used the place strategy significantly more often than PDC males $(P<$ $0.05, \chi^{2}=5.182$, Fig. 1B). Four of five PTC males used place strategy, whereas only one of seven PDC males used place strategy. Pre-exposure of animals to the same or different context prior to training did not affect the latency to escape the platform during training. Latency to find the platform during training trials revealed a significant effect of trial $\left(F_{(8,89)}=3.830, P=0.0007\right.$, non-repeated measures two-way ANOVA) but not pre-exposure condition $\left(F_{(1,89)}=0.103, P=0.75\right.$, non-repeated measures twoway ANOVA; Fig. 1C). Moreover, the average swim speed of PDC male mice $(6 \pm 1.6 \mathrm{~cm} / \mathrm{sec}, n=7)$ was not significantly different than the average swim speed of PTC male mice $(6 \pm 2.5 \mathrm{~cm} / \mathrm{sec}$, $n=5 ; P=0.34, t=0.9$ [t-test]). Together, these data suggest that the pre-exposure condition did not influence learning during the training period, but PTC did enhance place strategy use in the probe trial in male mice.

Female mice at 3 mo of age were randomly divided into two groups: mice that would receive ovariectomy (Ovx), and a sham surgery group (Sh). Mice were anesthetized with a ketamine $(80 \mathrm{mg} / \mathrm{kg})$ and xylazine $(8 \mathrm{mg} / \mathrm{kg})$ mixture. The first group of mice $(n=20)$ received ovariectomy using a dorsolateral approach. The other group $(n=19)$ of female mice received sham surgery, which consisted of ovary exposure only. Animals were injected with the pain reliever, buprenorphine $(5 \mathrm{mg} / \mathrm{kg})$, immediately after the surgery. One week after the surgery, vaginal smears were collected from all females, including Ovx as handled 
controls, at the same time each morning by lavage to track their estrus cycles (Marcondes et al. 2002). After two regular cycles, Sh animals were trained and tested on the day of proestrus (high estradiol).

Ovariectomy has been reported to affect anxiety levels (Walf et al. 2006), and anxiety levels may alter performance on water maze tasks. To assay possible anxiety differences between Sh and Ovx, female mice were tested on open field and elevated plusmaze (EPM) 2 wk after the surgery in a room different from the rooms used in water maze tasks. A single mouse was placed in the center of a white, Plexiglas chamber measuring $43 \mathrm{~cm}$ in length $\times 43 \mathrm{~cm}$ in width $\times 18 \mathrm{~cm}$ in height. The animal explored the novel environment for $15 \mathrm{~min}$, and movements were monitored by a camera interfaced with a tracking system (US HVS Image). The area was divided into 16 virtual squares $(10.75 \times$ $10.75 \mathrm{~cm}$ ) by the program, and the middle four squares were defined as the center area. The Plexiglas chamber was wiped clean with $70 \%$ ethanol between trials. The EPM consisted of four arms (5 $\mathrm{cm}$ in width $\times 30 \mathrm{~cm}$ in length) arranged perpendicularly in a plus shape and elevated $38 \mathrm{~cm}$ above the floor. Two arms were enclosed by $15.5-\mathrm{cm}$ dark Plexiglas walls and two arms were open. Each animal was placed in the center of the EPM facing a closed arm and allowed to move freely for $5 \mathrm{~min}$. Behavior was monitored by a camera interfaced with the tracking system.

Animals with high anxiety levels tend to spend less time in the open arms of the EPM and in the center of the open field. The percent time spent in the open arms of the EPM by Ovx mice $(37.9 \% \pm 7.5 \%, n=14)$ was not significantly different than the percent of time spent in the open arms by Sh mice $(27.8 \% \pm$ $6.2 \%, n=15 ; P=0.30, t=1.1)$. The percent time spent in the center of the open field by Ovx mice $(35.1 \% \pm 7.1 \%, n=14)$ was not significantly different from Sh mice $(29.0 \% \pm 7.1 \%, n=15 ; P=$ $0.55, t=0.61)$. These results indicate that ovarian hormones did not have a significant effect on the anxiety levels of the female mice tested in this study.

Two weeks after the anxiety tests, the Ovx and Sh groups were divided randomly into two groups based on the pre-exposure context: Ovx PTC, Ovx PDC, Sh PTC, Sh PDC. Sh females with regular estrus cycles were trained and tested on the day of proestrus. Five Ovx and seven Sh animals were not included in the study because of floating, failing to reach criterion (four correct out of nine trials), or exhibiting irregular estrus cycles. Five of eight $\mathrm{Sh}$ PTC and only one of six Sh PDC females used place strategy; however, this difference was not significant $\left(P>0.05, \chi^{2}=2.94\right.$, Fig. 2A). Therefore, the pre-exposure condition did not significantly affect strategy use in females at proestrus.

Interestingly, ovariectomy did significantly affect strategy use in PTC females. Five of eight Sh PTC and only one of eight Ovx PTC females used place strategy $\left(P<0.05, \chi^{2}=4.267\right.$, Fig. 2A). One of six Sh PDC females and zero of the six Ovx PDC animals used place strategy (Fig. 2A). Therefore, both Sh and Ovx PDC females used response strategy, and ovarian hormones did not enhance place strategy use in PDC females $(P>0.05$, $\chi^{2}=1.09$, Fig. 2A). Ovarian hormones did enhance place strategy use in PTC females. Furthermore, PTC did not enhance place strategy use in Ovx animals. Similar to males, there was a significant effect of training trial on latency to find the platform in female animals $\left(F_{(8,189)}=10.32, P<0.0001\right.$, Fig. 2B). Ovarian hormones or pre-exposure to either context also did not affect escape latency during training in PTC or PDC females $\left(F_{(3,189)}=\right.$ $0.33, P=0.80$, Fig. $2 \mathrm{~B})$. In addition, there was no significant difference in the average swim speed between groups $\left(F_{(3,14)}=0.15, P=\right.$ 0.93, one-way ANOVA). The average swim speed for each group was as follows: Ovx PTC $(5 \pm 1.5 \mathrm{~cm} / \mathrm{sec}, n=5)$, Ovx PDC $(5 \pm 1.0 \mathrm{~cm} / \mathrm{sec}, n=4)$, Sh PTC $(5 \pm 1.8 \mathrm{~cm} / \mathrm{sec}, n=5)$, Sh PDC $(6 \pm 1.5 \mathrm{~cm} / \mathrm{sec}, n=4)$. The numbers of animals are lower because
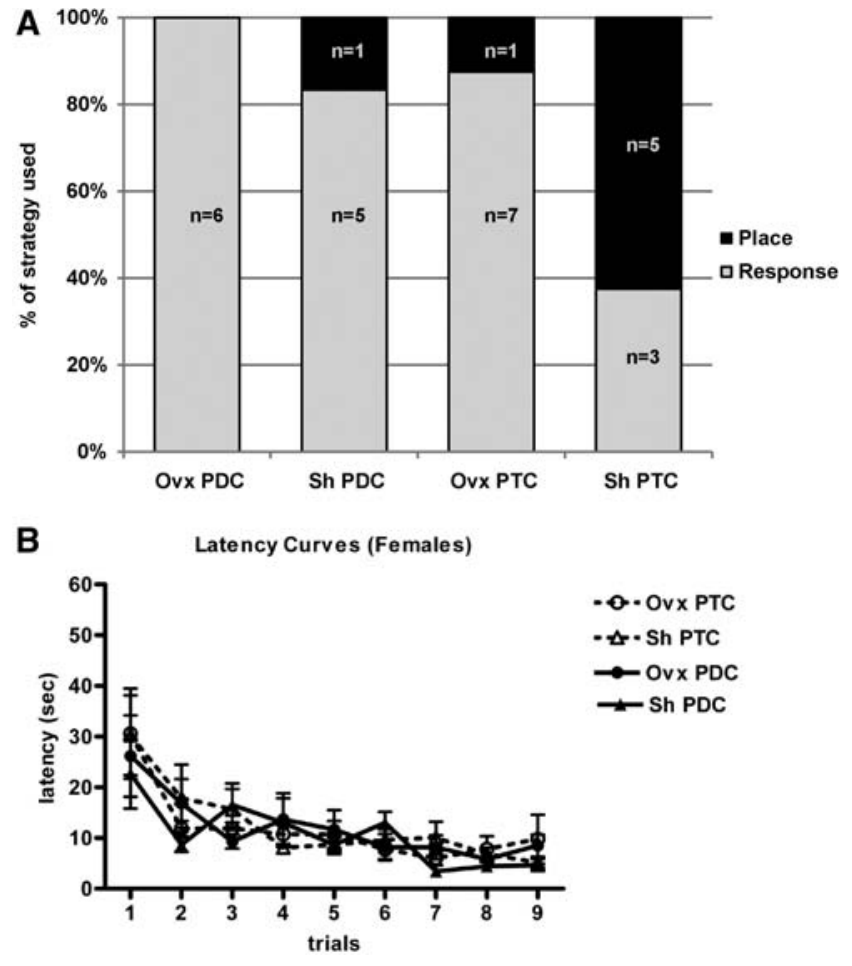

Figure 2. The effects of ovarian hormone status and pre-exposure to the training and testing (PTC) or to a different context (PDC) on strategy selection of female mice. $(A)$ When pre-exposed to the same context prior to training and testing (PTC), more gonadally intact female mice at proestrus (Sh, $n=8)$ used place strategy than response strategy compared with ovariectomized female mice (Ovx, $n=8, P<0.05$ ). When preexposed to a context different than the training and testing context (PDC), gonadally intact female mice at proestrus (Sh, $n=6)$ and ovariectomized mice $(\mathrm{Ovx}, n=6)$ used response strategy rather than place strategy. (B) Latency curves show the actual latency to escape to the platform. Two-way ANOVA (non-repeated measures) revealed no significant differences across training trials in escape latencies between sham and ovariectomized PTC and PDC mice $(P>0.5)$, although a significant effect of trial indicated that mice reduced their escape latencies across trials $(P<0.0001)$. Values represent mean \pm S.E.M.

in some cases, speed was not measured. Together, these data suggest that ovarian hormones and pre-exposure condition did not influence learning during the training period, but ovarian hormones did enhance place strategy use in the probe trial in only PTC mice.

Consistent with previous literature (Martel et al. 2007), we found that $\sim 80 \%$ of PTC males favored the use of place strategy. In addition, $63 \%$ of PTC females on proestrus also used place strategy. Ovx female mice used response strategy regardless of the preexposure condition. These results confirm that pre-exposure to the training and testing context significantly increased the use of place strategy or reduced response strategy in male mice, while female mice on proestrus were not significantly different than chance. Ovariectomy diminished the use of place strategy and enhanced response strategy use in our study, implicating ovarian hormones in strategy choice.

Male rats rely initially on a hippocampus-dependent place strategy, and then switch to a striatum-based response strategy over training (Packard and McGaugh 1996; Packard 1999). This suggests that response strategy is incrementally learned with repeated exposure to the same task. However, a sufficient amount of time to explore the extramaze cues during or before training increased place strategy use in male mice (Martel et al. 2007). 
In addition, it has been proposed that the presence of an increased number of salient extramaze cues favors place strategy use in rats (Restle 1957). Therefore, it is possible that pre-exposing mice to the learning environment allowed them to build a cognitive map that facilitated the use of a spatial place strategy. Another possible advantage of pre-exposure for place strategy use is that it may reduce the impact of non-mnemonic factors, such as anxiety, on performance (Cain 1998). Indeed, it was shown that peripheral injection and infusion of anxiogenic drugs into the basolateral amygdala biased rats toward the use of response strategy (Packard 1999; Wingard and Packard 2008; Packard and Gabriele 2009).

While PTC female mice were not significantly different than PDC female mice, ovariectomy did reduce place strategy choice in the PTC mice. An emerging theory proposes that estradiol modulates cognitive performance via shifts in learning strategy (Korol and Kolo 2002; Daniel and Lee 2004; Korol 2004; McElroy and Korol 2005; Zurkovsky et al. 2007). Shifts in strategy use occurred across the estrus cycle in rats such that the hippocampus-dependent strategy was favored when estradiol levels were high (Korol et al. 2004). Similarly, estradiol treatment in ovariectomized rats increased hippocampus-dependent place strategy and impaired response strategy use compared with nontreated ovariectomized females (Korol and Kolo 2002). Our results showing that the lack of ovarian hormones reduced place strategy and increased response strategy use in PTC mice are consistent with these studies.

In summary, we present a new design to a traditional dualsolution land plus-maze. One issue with the land maze version of the task is that it requires food deprivation. The possible increase in the appetite as a result of ovariectomy (Wade 1975) or disruption in the estrus cycle in response to food deprivation (Daniel et al. 1999) could confound the results in females in tasks that present food reward. In order to avoid these confounds, we used a modified version of a water-escape plus-maze (Packard and Wingard 2004). In this design, compared with the waterescape plus-maze, the clear Plexiglas maze itself is filled with water, instead of placing the plus-maze into a water maze, allowing a better view of extramaze visual cues. However, unlike rats, mice tend to be prey animals when in the water; therefore they are highly motivated to escape the water (Francis et al. 1995; Van Dam et al. 2006). Consequently, the stressful nature of the task prevents mice from utilizing the spatial cues as efficiently (Frick et al. 2000). Therefore, we pre-exposed the mice to the maze while it was dry, allowing them to build a cognitive map before they were released in water. The water plus-maze is important not only for the design of future studies, but also for the evaluation of previous studies that investigated learning strategies using tasks dependent on food deprivation.

\section{Acknowledgments}

This work was supported by NIH/COBRE grant P20RR016816: Mentoring Neuroscience in Louisiana. We thank Dan Liu and Claire Stelly for technical support, and Jill Daniel and Johannes Bohacek for helpful advice.

\section{References}

Cain DP. 1998. Testing the NMDA, long-term potentiation, and cholinergic hypotheses of spatial learning. Neurosci Biobehav Rev 22: 181-193.

Daniel JM, Lee CD. 2004. Estrogen replacement in ovariectomized rats affects strategy selection in the Morris water maze. Neurobiol Learn Mem 82: $142-149$.

Daniel JM, Roberts SL, Dohanich GP. 1999. Effects of ovarian hormones and environment on radial maze and water maze performance of female rats. Physiol Behav 66: 11-20.
Dohanich GP. 2002. Gonadal steroids, learning, and memory. In Hormones, brain and behavior, Vol. 2 (ed. DW Pfaff et al.), pp. 265-327. Academic Press, San Diego, CA.

Dohanich GP, Korol D, Shors T. 2009. Steroids, learning, and memory. In Hormones, brain and behavior, 2nd ed., Vol. 1 (ed. DW Pfaff et al.), pp. 265-327. Academic Press, San Diego, CA.

Francis DD, Zaharia MD, Shanks N, Anisman H. 1995. Stress-induced disturbances in Morris water-maze performance: Interstrain variability. Physiol Behav 58: 57-65.

Frick KM, Stillner ET, Berger-Sweeney J. 2000. Mice are not little rats: Species differences in a one-day water maze task. Neuroreport 11: $3461-3465$.

Gold PE. 2004. Coordination of multiple memory systems. Neurobiol Learn Mem 82: 230-242.

Korol DL. 2004. Role of estrogen in balancing contributions from multiple memory systems. Neurobiol Learn Mem 82: 309-323.

Korol DL, Kolo LL. 2002. Estrogen-induced changes in place and response learning in young adult female rats. Behav Neurosci 116: $411-420$.

Korol DL, Malin EL, Borden KA, Busby RA, Couper-Leo J. 2004. Shifts in preferred learning strategy across the estrous cycle in female rats. Horm Behav 45: 330-338.

Lee AS, Duman RS, Pittenger C. 2008. A double dissociation revealing bidirectional competition between striatum and hippocampus during learning. Proc Natl Acad Sci 105: 17163-17168.

Marcondes FK, Bianchi FJ, Tanno AP. 2002. Determination of the estrous cycle phases of rats: Some helpful considerations. Braz J Biol 62: 609-614.

Martel G, Blanchard J, Mons N, Gastambide F, Micheau J, Guillou JL. 2007. Dynamic interplays between memory systems depend on practice: The hippocampus is not always the first to provide solution. Neuroscience 150: $743-753$.

McElroy MW, Korol DL. 2005. Intrahippocampal muscimol shifts learning strategy in gonadally intact young adult female rats. Learn Mem 12: 150-158.

Mizumori SJY, Yeshenko O, Gill KM, Davis DM. 2004. Parallel processing across neural systems: Implications for a multiple memory system hypothesis. Neurobiol Learn Mem 82: 278-298.

Packard MG. 1999. Glutamate infused post training into the hippocampus or caudate-putamen differentially strengthens place and response learning. Proc Natl Acad Sci 96: 12881-12886.

Packard MG, Gabriele A. 2009. Peripheral anxiogenic drug injections differentially affect cognitive and habit memory: Role of basolateral amygdala. Neuroscience 164: 457-462.

Packard MG, McGaugh JL. 1992. Double dissociation of fornix and caudate nucleus lesions on acquisition of two water maze tasks: Further evidence for multiple memory systems. Behav Neurosci 106: $439-446$.

Packard MG, McGaugh JL. 1996. Inactivation of hippocampus or caudate nucleus with lidocaine differentially affects expression of place and response learning. Neurobiol Learn Mem 65: 65-72.

Packard MG, Teather LA. 1997. Intra-hippocampal estradiol infusion enhances memory in ovariectomized rats. Neuroreport 8: 3009-3013.

Packard MG, Wingard JC. 2004. Amygdala and 'emotional' modulation of the relative use of multiple memory systems. Neurobiol Learn Mem 82: 243-252.

Restle F. 1957. Discrimination of cues in mazes: A resolution of the place-vs.-response question. Psychol Rev 64: 217-228.

Van Dam D, Lenders G, De Deyn PP. 2006. Effect of Morris water maze diameter on visual-spatial learning in different mouse strains. Neurobiol Learn Mem 85: 164-172.

Wade GN. 1975. Some effects of ovarian hormones on food intake and body weight in female rats. J Comp Physiol Psychol 88: 183-193.

Walf AA, Rhodes ME, Frye CA. 2006. Ovarian steroids enhance object recognition in naturally cycling and ovariectomized, hormone-primed rats. Neurobiol Learn Mem 86: 35-46.

White NM, McDonald RJ. 2002. Multiple parallel memory systems in the brain of the rat. Neurobiol Learn Mem 77: 125-184.

Wingard JC, Packard MG. 2008. The amygdala and emotional modulation of competition between cognitive and habit memory. Behav Brain Res 193: $126-131$.

Wolfer DP, Müller U, Stagliar M, Lipp HP. 1997. Assessing the effects of the 129 /Sv genetic background on swimming navigation learning in transgenic mutants: A study using mice with a modified $\beta$-amyloid precursor protein gene. Brain Res 771: 1-13.

Zurkovsky L, Brown SL, Boyd SE, Fell JA, Korol DL. 2007. Estrogen modulates learning in female rats by acting directly at distinct memory systems. Neuroscience 144: 26-37.

Received March 26, 2010; accepted in revised form May 12, 2010. 


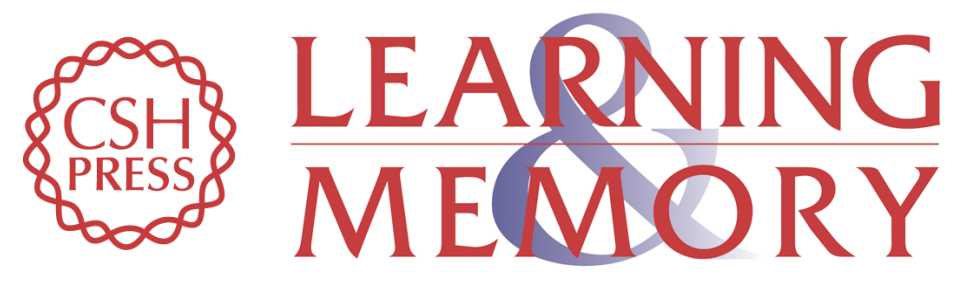

\section{Pre-exposure to context affects learning strategy selection in mice}

Tumay Tunur, Gary P. Dohanich and Laura A. Schrader

Learn. Mem. 2010, 17:

Access the most recent version at doi:10.1101//m.1818410

References This article cites 30 articles, 3 of which can be accessed free at: http://learnmem.cshlp.org/content/17/7/328.full.html\#ref-list-1

License

Email Alerting Receive free email alerts when new articles cite this article - sign up in the box at the Service top right corner of the article or click here. 